\title{
La New Haven School: Una breve introducción
}

\author{
The New Haven School: A Brief Introduction
}

\author{
W. Michael Reisman \\ michael.reisman@yale.edu \\ Doctor en Derecho (J.S.D) por la Universidad de Yale (1965). Profesor de Derecho Internacional en la \\ Cátedra Myres S. McDougal, Escuela de Derecho de la Universidad de Yale (Estados Unidos).
}

\section{Siegfried Wiessner}

swiessner@stu.edu

Doctor en Derecho (Dr. iur.) por la Universidad de Tubinga, Alemania (1989). Profesor de Derecho, Fundador y Director del Programa de Graduados en Derechos Humanos Interculturales, Escuela de Derecho de la Universidad de Saint Thomas (Estados Unidos).

\section{Andrew R. Willard}

andrew-willard@uiowa.edu

Antropólogo. Coordinador de Enseñanza Experimental, Programas Universitarios de Honor, Universidad de Iowa. Investigador Senior en la Escuela de Derecho de la Universidad de Yale (Estados Unidos).

Nota del Editor: El presente artículo es una traducción y adaptación, aprobada por sus autores, de un texto aparecido en el The Yale Journal of International Law. Nos ha parecido de interés su publicación a fin de incentivar la discusión sobre las metodologías de análisis jurídico internacional. La New Haven School plantea una aproximación teleológica al derecho internacional (y eventualmente también nacional) a fin de superar las deficiencias del realismo legal norteamericano y como una reacción frente a la pretendida neutralidad, formalismo y enfoque meramente descriptivo del positivismo. El sistema jurídico es visto como un "proceso de toma de decisiones" y no como algo estático. Al desplazar la atención desde las normas formales al modo en que las decisiones son efectivamente tomadas, adquieren especial relevancia los valores a los que da preeminencia el sistema internacional, así como la interrelación entre el derecho 
internacional y otros procesos mundiales, sociales o económicos. Ello provoca, sin embargo, un alto grado de indeterminación en el derecho, perdiendo este su autonomía e independencia. De aquí han surgido las principales críticas a este enfoque. Pero sus defensores, próximos al realismo al fin y al cabo, arguyen que eso es lo que efectivamente ocurre en la práctica, debiendo ser así asumido. Esperamos incentivar la discusión académica al respecto.

Con el debido respeto al ágora y a los peripatéticos, una "escuela del pensamiento" es más que un mero punto de encuentro. Ella implica una comunidad de académicos que se identifica como tal, comparte ideas sobre el proceso social, un mismo sentido de misión respecto a dicho proceso y una metodología común. Como se ha demostrado en encuentros académicos recientes, eso es lo que ocurre con la New Haven School.

La New Haven School fue desarrollada por los Profesores Myres S. McDougal y Harold D. Lasswell en la Escuela de Derecho de la Universidad de Yale ${ }^{1}$. McDougal había estudiado cultura clásica y luego, en Oxford, historia del derecho. Lasswell, en el período que conoció a McDougal, ya era considerado uno de los cientistas políticos y sociales más creativos del siglo XX. La escuela de pensamiento jurídico que crearon en Yale adapta los métodos analíticos de las ciencias sociales a los propósitos regulatorios del derecho. Aplicando múltiples instrumentos, busca desarrollar herramientas para producir cambios en el orden público y en el sistema social que permitan aproximarse a los objetivos de dignidad humana que ella postula.

La New Haven School define al derecho como un proceso de decisión que es tanto de autoridad (legitimidad) como de control (efectividad); sitúa las decisiones pasadas bajo el enfoque de los factores que las condicionaron, ambientales y predisposicionales; evalúa las tendencias decisionales de acuerdo a su compatibilidad con los objetivos declarados; pronostica, tanto como sea posible, las futuras alternativas de decisión y sus consecuencias; y provee de herramientas conceptuales para aquellos que las requieren para crear y evaluar decisiones alternativas, acuerdos constitutivos y cursos de acción orientados por un ideal que privilegie un

1 El trabajo más relevante de la New Haven School es el tratado en dos volúmenes de Myres S. McDougal y Harold D. Lasswell, Jurisprudence for a Free Society: Studies in Law, Science and Policy (New Haven: New Haven Press, 1992). Trabajos anteriores a ese fueron Harold D. Lasswell, World Politics and Personal Insecurity (NuevaYork: Whittlesey House, McGrawHill Book Co., 1935); Myres S. McDougal y Harold D. Lasswell, "Legal Education and Public Policy: Professional Training in the Public Interest", The Yale Law Journal, 52(2) (1943) 203-295; Harold D. Lasswell y Abraham Kaplan, Power and Society: A Framework for Political Inquiry (New Haven: Yale University Press, 1950); Myres S. McDougal, "International Law. Power and Policy: A Contemporary Conception", Collected courses of The Hague Academy of International Law (Recueil des cours de l'Académie de droit international de La Haye), 82 (1953) 133-260; Myres S. McDougal, Harold D. Lasswell y W. Michael Reisman, "Theories about International Law: Prologue to a Configurative Jurisprudence", Virginia Journal of International Law, 8(2) (1968) 188-299; Myres S. McDougal, W. Michael Reisman y Andrew R. Willard, "The World Community: A Planetary Social Process", U.C. Davis Law Review, 21 (1988) 807-972. Otros ejemplos de esta aproximación metodológica incluyen W. Michael Reisman, "Theory about Law: The New Haven School of Jurisprudence”, Wissenschaftskolleg Jahrbuch (1989-1990) 228-242; y, Siegfried Wiessner y Andrew R. Willard, "Policy-Oriented Jurisprudence", German Yearbook of International Law, 44 (2001) 96-112. De modo alternativo, sus principales cultores han designado esta metodología como "ciencia del derecho políticamente-orientada" (policy-oriented jurisprudence) o "derecho, ciencia y política". 
orden público centrado en la dignidad humana. Para lograr estos objetivos, la New Haven School adapta el enfoque de las ciencias sociales, proponiendo una manera de organizar los datos de varios procesos sociales mediante un análisis de fases y un desglose analítico de los componentes efectivos de una decisión, tal como lo hace la antropología cultural. A fin de facilitar la toma de decisiones, la New Haven School propone cinco tareas intelectuales: formulación de objetivos, descripción de tendencias, análisis de factores, proyección de decisiones futuras y elaboración de alternativas. Un orden público centrado en la dignidad humana se define como aquel que se aproxima al óptimo acceso para todos los seres humanos a todo lo que ellos aprecian: poder, riqueza, ilustración, habilidades, bienestar, afecto, respeto y rectitud. Esto, en resumen, caracteriza la contribución que la New Haven School ha hecho a la enseñanza del derecho y a la actividad política.

Debería ser evidente que las herramientas intelectuales de la New Haven School son útiles para cualquier persona que esté empeñada en resolver un problema, cualquiera sea el contexto. Pero vale la pena enfatizar este punto, porque mucha gente la asocia únicamente con el derecho y la política internacional ${ }^{2}$. Esa asociación es entendible a la luz de su concepción del ámbito planetario como "un enorme colector", en palabras de Whitehead", y debido a la extensa literatura que ha aplicado la metodología de la New Haven School a problemas internacionales. En realidad, estos métodos y procedimientos están diseñados y han sido usados para entender y conformar el derecho en todos los contextos, desde lo local a lo global.

Sin perjuicio de ello, la New Haven School es útil, en especial, para los individuos que no están vinculados con el Estado, los que constituyen un grupo al que el derecho internacional clásico había privado de sus derechos políticos. En el pasado, el cliente del abogado internacionalista era, mayoritariamente, el Príncipe o, puesto en términos más generales, los gobiernos, como fuera que ellos estuvieran organizados. Ya no es así. Equipada con un marco de conocimientos jurídicos adecuado, cada una y todas las personas pueden participar, directamente o mediante agrupaciones, en los procesos de decisión que afectan sus vidas. En este sentido, todos tenemos el potencial de desempeñarnos como el Príncipe o como el "superior político" de Austin ${ }^{4}$. La constante formación y reformulación de grupos de interés en un planeta en el que la simultaneidad económica-electrónica permite una coordinación efectiva sin requerir de un contacto personal directo, implica que la posibilidad de tener una participación significativa en las funciones claves de la toma de decisiones internacionales está al alcance de cada vez más personas que no son parte de los gobiernos. Pero solo si saben cómo hacerlo.

2 Curiosamente, fue un académico europeo quien en un estudio sobe la New Haven School concluyó que sus métodos eran más efectivos para los problemas jurídicos de derecho doméstico. Vid. Bent Rosenthal, Etude de l'oeuvre de Myres Smith McDougal en matières de droit international public (Paris: Pichon et Durand-Auzias, 1970).

3 Alfred North Whitehead, Science and the Modern World (1941). Véase también Alfred North Whitehead, Process and Reality: An Essay in Cosmology (Nueva York: The Free Press, 1978).

4 W. Michael Reisman, “A Jurisprudence for the Perspective of the 'Political Superior”, Northern Kentucky Law Review, 23(3) (1996) 605-627. 
La New Haven School: Una breve introducción

Para todos estos actores, la New Haven School reúne un conjunto de herramientas destinadas a mejorar la comprensión y aumentar su influencia efectiva en dichos procesos internacionales. Es una obviedad decir que el derecho es político, pero esta es una aproximación políticamente orientada en un sentido mucho más amplio. Con respecto a problemas particulares, no solamente se buscan visualizar los procesos de decisión, evaluar las tendencias muchas veces contradictorias y los factores que los condicionan, predecir el rango de probables resultados y mejorar las habilidades necesarias para influir en el proceso de decisión de modo de alcanzar los resultados esperados. También se busca mejorar los procesos de decisión en sí mismos y aumentar su capacidad de generar resultados acordes con la dignidad humana. Esto necesariamente incluye una evaluación cuidadosa y crítica a los actuales procesos, instituciones y prácticas.

Es claro que, dadas las características de los procesos políticos internacionales, una vía para visualizar los desarrollos en los que los abogados tratan de influir y que a su vez influyen en los abogados, requiere de un conjunto especial de herramientas analíticas. Un análisis "convencional", en términos de los órganos del gobierno y de las técnicas empleadas por los funcionarios estatales, aunque fuera efectivo para ciertos problemas, resulta inapropiado para el estudio de las decisiones internacionales. El modo convencional debe ceder ante un análisis "funcional", ya que en la escala global no existe una relación de dependencia entre las estructuras formales y los hechos de autoridad y control. No es para nada inusual descubrir, por ejemplo, que la autoridad formalmente señalada en una norma escrita puede ser ignorada o totalmente redefinida por una práctica no-escrita; debe también distinguirse entre un sistema mítico y un código operacional ${ }^{5}$, entre el derecho-en-los-libros y el derecho-en-acción. Cuando se examina el ámbito internacional, la presumida congruencia entre la autoridad formal y la autoridad real puede contar o no con la concurrencia de las expectativas necesarias que justifiquen la pretensión de la autoridad constituida.

En consecuencia, el marco comprehensivo y analítico requerido debe incluir una técnica conceptual para visualizar los procesos relevantes. La New Haven School ha adaptado, con varios ajustes, un esquema de la antropología cultural, en el cual cualquier proceso social es descrito sistemáticamente en términos de quienes participan en él (participantes); las dimensiones subjetivas que los animan (sus perspectivas); las situaciones en cuales ellos interactúan (situaciones); los recursos sobre los cuales ellos se sustentan (bases de poder); las formas como ellos utilizan esos recursos (estrategias); y los resultados agregados de los procesos de interacción (resultados), que son concebidos como un conjunto amplio de valores.

Los participantes en cualquier proceso de decisión incluyen a aquellos formalmente dotados con competencia para decidir, tal como funcionarios públicos, legisladores y jueces, y a todos aquellos otros actores que, aunque no estén dotados de competencias formales, pueden influir en forma significativa en la decisión final. Respecto a las decisiones internacionales, eso

5 W. Michael Reisman, Folded Lies: Bribery, Crusades and Reform (Nueva York: The Free Press, 1979). 
significa examinar, además de las organizaciones internacionales formales, a los funcionarios de los Estados ${ }^{6}$, a las organizaciones no gubernamentales $(\mathrm{ONG})^{7}$, y a los grupos de presión, grupos de interés, agrupaciones e individuos, los que actúan tanto en nombre de otros participantes como por sí mismos.

De igual forma, este inventario no sería muy útil si no se toma en cuenta la perspectiva desde la cual actúan estos actores. Estas perspectivas incluyen sus patrones específicos de identificación y de desafección, sus expectativas objetivas sobre el pasado y el futuro, y las demandas valóricas que ellos proyectan. Es claro que en un ámbito tan complejo como lo es el de la política internacional, las perspectivas de los diferentes participantes que tienen un papel en la decisión divergen muchas veces de un modo crítico.

Las situaciones, en el sentido que la New Haven School da a este término, se refieren generalmente a los lugares donde se toman las decisiones, y las propiedades distintivas de ese "dónde". El análisis legal convencional generalmente mira hacia las cortes, y secundariamente examina el trabajo del poder ejecutivo y de la legislatura. La New Haven School adopta una aproximación más funcional, de acuerdo a la cual se enfoca en las instancias centralizadas o descentralizadas ${ }^{9}$ en cuales se toman realmente las decisiones, en su variado grado de organización y formalidad, en hasta qué punto ellas son o no especializadas, y si son situaciones continuas o episódicas. Consideramos además importante examinar en qué nivel los participantes en un hecho en particular se perciben a sí mismos como sufriendo un estado de crisis en el cual sus valores críticos están en juego.

Los recursos en los cuales los participantes se sustentan -sus bases de poder- incluyen tanto su poder efectivo como sus símbolos de autoridad. La New Haven School considera apropiado que el jurista correlacione hasta qué punto es posible controlar el poder a fin de sustentar las formulaciones particulares que se presentan como derecho.

La forma en que se manejan los recursos (materiales y simbólicos) o las estrategias usadas por los diferentes participantes, involucra la gestión de los mismos a fin de optimizar los resultados esperados. Las modalidades estratégicas son consideradas como partes de un continuum persuasivo-coactivo. Ellas consideran las técnicas diplomáticas, propagandísticas, económicas y militares, en distintas proporciones.

6 Mientras los Estados continúan siendo las formas de organización política primaria y los principales proveedores de valores en sus sociedades, las entidades privadas, con su efectividad aumentada por las innovaciones tecnológicas relativamente libres del control por parte de las elites territoriales (como el Internet), ejercen una influencia cada vez mayor en el proceso global de decisiones de autoridad y control. Vid. W. Michael Reisman, "Designing and Managing the Future of the State", European Journal of International Law, 8(3) (1997) 416.

7 Siegfried Wiessner, "Legitimacy and Accountability of NGOs: A Policy-Oriented Perspective", en W. Michael Reisman et al., International Law in Contemporary Perspective, $2^{\mathrm{a}}$ ed. (Nueva York: Foundation Press, 2004) 305-311.

$8 \quad$ McDougal, Reisman y Willard, “The World Community...”, 822-826.

9 Respecto a la elección del marco metodológico dependiendo de las determinantes institucionales, vid. Siegfried Wiessner, "International Law in the $21^{\text {th }}$ Century: Decision-Making in Institutionalized and Non-Institutionalized Settings", Thesaurus Acroasium, 26 (1997) 129. 
Los resultados de la interacción son expuestos en una base continua y en términos de las cinco fases o características de un contexto que fueron identificadas anteriormente.

La visualización de los contextos sociales y de decisión permite al observador o a quien trata de influir en tales decisiones orientarse dentro de los procesos relevantes. Es evidente que no puede existir una sola y única solución para todo tipo de problema. Los contextos varían enormemente. Por lo tanto, los participantes que serán relevantes para un problema que involucra la seguridad internacional, por ejemplo, incluirán a las grandes potencias, a aquellos que pueden apoyarlas o actuar en su contra, incluyendo a sus mayores antagonistas, a las organizaciones internacionales y a otras instituciones que pudieran ser relevantes para algunas decisiones. Por su parte, para un problema que concierne al derecho de la inversión extranjera en un contexto específico, los participantes críticos serán probablemente los Estados involucrados, las empresas multinacionales afectadas, la sociedad civil y las instituciones especializadas en procesos económicos transnacionales. El punto a enfatizar es que un enfoque funcional, usando un procedimiento de visualización diseñado para minimizar la probabilidad de pasar por alto los factores y relaciones pertinentes, permite al abogado y al cientista político operar con un sentido realista acerca de los procesos relevantes que enfrenta.

La ciencia moderna ha sido muy consciente de la necesidad de tomar en cuenta y tener claridad sobre la perspectiva desde la cual los fenómenos son observados. Para cualquier fenómeno hay varios puntos de vistas posibles, cada uno de los cuales afecta y da forma a lo que se ve y cómo se lo ve. Una herramienta intelectual indispensable consiste en poseer claridad respecto a lo que la New Haven School ha denominado el "punto de vista observacional"10. La referencia y el contenido del término "derecho" o "elección”, por ejemplo, variarán dependiendo de si el punto de vista es el de un miembro de la élite o el de un hombre común, y de si el observador es parte del sistema observado y ha internalizado ya sus costumbres, mitos y modos característicos o es una persona ajena a tal sistema. La percepción de un mismo fenómeno puede variar según la cultura, clase social, género, edad o, en particular, la influencia profunda de las experiencias críticas o traumas del observador. Incluso dentro de las instituciones legales formales, la referencia y el contenido del derecho variarán dependiendo de si el observador es un legislador, juez, fiscal, jurado, defensor, acusado o víctima.

La delimitación del enfoque apropiado es importante porque afecta la exhaustividad y el realismo (o la apreciación del contexto) de la investigación, la aproximación práctica desde la cual los problemas son formulados, y la efectividad con que las diferentes tareas intelectuales pueden ser realizadas. En este sentido, el derecho es concebido no simplemente como un conjunto de reglas, a la manera tradicional ${ }^{11}$, sino en términos más comprehensivo, como una

10 W. Michael Reisman, “The View from New Haven School of International Law”, American Society of International Law Proceedings, 86 (1992) 120.

11 "La aproximación al derecho que hace el positivismo entrega la imagen opuesta de esta concepción empírica y dinámica del derecho. Su enfoque en las reglas 'existentes', emanadas únicamente desde entidades estimadas como igualmente 'soberanas', no refleja adecuadamente la realidad de cómo el derecho es creado, aplicado y modificado. El positivismo permanece anclado en el pasado, tratando de obtener desde las palabras, y sin considerar el contexto en que ellas fueron 
decisión compuesta al mismo tiempo de perspectivas y de operaciones; como una decisión imperativa, combinando elementos de autoridad y de control; y no como una determinación ocasional, sino como un proceso continuo de toma de decisiones, que a la vez que mantiene las características constitutivas por las cuales los valores de la comunidad fueron establecidos, se proyecta hacia el futuro en un flujo de decisiones de orden público destinadas a formar y compartir tales valores ${ }^{12}$.

Un componente de este proceso, así como de todos los procesos básicos de una comunidad, es que él represente un poder efectivo, en el sentido de que las decisiones son realmente tomadas y hechas cumplir, ya sea mediante castigos severos o a través de grandes indulgencias, los que son inclusivos en sus efectos. La experiencia de aquellos que usan la metodología de la New Haven School demuestra que una descripción completa y realista de tales procesos de poder efectivo -en términos que incluya a todos los participantes, perspectivas, ámbitos, bases de poder, estrategias y resultados relevantes- es necesaria para entenderlos e influir en ellos.

La New Haven School también provee de una forma de organizar las tareas intelectuales y prácticas requeridas en cualquier proceso de decisión. Se identifican siete funciones, cada una de las cuales está involucrada en cada proceso decisorio. Ellas son: inteligencia (obtención de información), promoción (de los programas o cambios deseados), prescripción (dar mandatos imperativos), invocación (del respeto a los mandatos dados), aplicación (de los mandatos en una situación en particular), terminación (de los efectos imperativos de aquellos mandatos que ya no reflejan apropiadamente los objetivos del orden público deseado) y evaluación (acerca de la forma en que se desarrolló el proceso de decisión, su efectividad y su eficiencia) ${ }^{13}$. El hecho que cada función esté operando no significa que esté siendo bien ejecutada. En consecuencia, la New Haven School ofrece también criterios para evaluar el desempeño de cada función y, al aportar claridad respecto a ellas, se provee de una vía realista y equilibrada para mejorar la decisión.

Partiendo de la premisa que el derecho debiera servir a los seres humanos, la New Haven School fija su búsqueda políticamente-orientada de un orden público mundial en favor de la dignidad humana ${ }^{14}$ dentro del universo de las aspiraciones de las personas, las cuales son expresadas

escritas, la solución a los problemas que surgen hoy día o surgirán mañana en unas circunstancias muy distintas". Vid. Siegfried Wiessner y Andrew R. Willard, "Policy-Oriented Jurisprudence and Human Rights Abuses in Internal Conflict: Toward a World Public Order of Human Dignity", American Journal of International Law, 93 (1999) 320. Respecto a la visión de otras aproximaciones jurídicas, ver el trabajo citado, $320 \mathrm{n} .7$.

12 Myres S. McDougal, Harold D. Lasswell y W. Michael Reisman, “The World Constitutive Process of Authoritative Decision", Journal of Legal Education, 19 (1967) 253; reimpreso en Cyril E. Black y Richard A. Falk, edit., The Future of the International Legal Order. Trends and Patterns (New Jersey: Princeton University Press, 1969), vol. 1, 73; también reimpreso en Myres S. McDougal y W. Michael Reisman, International Law Essays: A Supplement to International Law in Contemporary Perspective (Nueva York: Foundation Press, 1981), 191.

13 McDougal, Lasswell y Reisman, “The World Constitutive Process...”; véase también, Reisman, "The View from New Haven School...”, 122-123.

14 Cfr. Myres S. McDougal, Harold D. Lasswell y Lung-Chu Chen, Human Rights and World Public Order: The Basic Policies of an International Law of Human Dignity (New Haven, Yale University Press, 1980). Véase también Wiessner y Willard, "PolicyOriented Jurisprudence and Human Rights Abuses...". 
La New Haven School: Una breve introducción

W. Michael Reisman, Siegfried Wiessner y Andrew R. Willard

empíricamente en ocho valores ${ }^{15}$, a saber: poder, ilustración, riqueza, bienestar, habilidades, afecto, respeto y rectitud. La metodología propuesta permite definir objetivos que vayan más allá de lo que ya se ha logrado en el pasado y preparar intelectualmente a los líderes para enfrentar los siempre-nuevos problemas que aparecen en un mundo en cambio permanente.

15 El término "valores" es usado para designar la amplia categoría de eventos que gratifican los deseos. En términos empíricos, ellos significan las cosas que las personas "valoran". Analíticamente, ellos caracterizan los activos a los que las personas recurren para lograr sus objetivos; desde la perspectiva de las preferencias, ellos se refieren a los "eventos preferidos". Vid. McDougal y Lasswell, Jurisprudence for a Free Society..., 336. 